\title{
The clinical evaluation of Vi-one chlorhexidine mouthwash on plaque-induced gingivitis: A double-blind randomized clinical trial
}

\author{
Babak Amoian ${ }^{1}$, Marzyeh Omidbakhsh², Soraya Khafri ${ }^{3}$
}

${ }^{1}$ DDS., MSc. In Periodontology, Associate Professor, Department of Periodontics, Faculty of Dentistry, Babol University of Medical Sciences, Babol, Iran

${ }^{2}$ Dental Student, Faculty of Dentistry, Babol University of Medical Sciences, Babol, Iran

${ }^{3}$ Ph.D. of Assistant Professor, Department of Statistic and Epidemiology, Faculty of Medicine, Babol University of Medical Sciences, Babol, Iran

\section{Type of article: Original}

\begin{abstract}
Background: Chlorhexidine (CHX) is the most effective antiseptic mouthwash to date. Essential oil such as thymol, have inhibitory and biocidal effects a range of bacteria.

Objective: To determine the effect of mouthwash containing CHX and thymol on plaque induced gingivitis.

Methods: This double-blind randomized clinical trial study was performed on 60 patients with plaque induced gingivitis who were randomly divided into two groups: Group I (CHX/thymol mouthwash-Vi-one) and Group II (CHX mouthwash-Behsa). Patients in each group underwent scaling and root planning and polishing, then were educated about BASS-Method brushing, and were recommended Oral-B toothbrushes and Pooneh toothpaste. The two groups were asked to rinse their mouths for 60 seconds twice a day, once in the morning and once at night, after brushing their teeth. Plaque index, gingival index, bleeding index and stain index were evaluated at baseline and 14 days later in Ramfjord teeth. Data analysis was conducted using SPSS version 21. Independentsamples t-test and paired-samples t-test were used for data analysis.

Results: The results showed that plaque index and gingival index significantly reduced in two groups $(\mathrm{p}<0.001)$. However, group I was significantly more efficient than group II $(\mathrm{p}<0.001, \mathrm{p}=0.021$ respectively). Similar results were observed in terms of bleeding index with the difference that the two groups did not differ significantly from each other $(\mathrm{p}=0.879)$. Both groups significantly increased the stain index. No remarkable difference was also observed between the two groups ( $\mathrm{p}=0.754)$.

Conclusion: Based on the results of this study, we can conclude that the CHX/thymol mouthwash can be offered to patients with dental plaque-induced gingivitis, because it appears to be more effective in controlling dental plaque and gingivitis.

Trial registration: The trial was registered at the Iranian Registry of Clinical Trials (http://www.irct.ir) with the Irct ID: IRCT201602231760N45.

Funding: This study was part of a thesis and research project (Grant No: 9440921) supported and funded by Babol University of Medical Sciences. The authors received no financial support from Behsa pharmaceutical company (Behsa mouthwash manufacturer) or Rojin cosmetic laboratory (Vi-one mouthwash manufacturer) for this study.
\end{abstract}

Keywords: Mouthwashes, Chlorhexidine, Gingivitis, Periodontal index, Thymol

\section{Introduction}

Dental plaque is a sticky and soft biofilm that is formed on the tooth surface $(1,2)$. It begins to form on tooth surfaces within only a few minutes after brushing the teeth. If the dental plaque is not eliminated, it can result in dental caries and periodontal diseases such as gingivitis and periodontitis (1). Periodontal diseases affect the toothsupporting tissues; gingivitis is the mildest form of periodontal disease, which generally develops due to inadequate

\section{Corresponding author:}

Marzyeh Omidbakhsh, Faculty of Dentistry, Babol University of Medical Sciences, Babol, Iran.

Tel: +98.9117717142, Email: MarzyehOmidbakhsh@yahoo.com

Received: August 13, 2016, Accepted: September 23, 2016, Published: September 2017

iThenticate screening: September 21, 2016, English editing: April 01, 2017, Quality control: May 02, 2017

(C) 2017 The Authors. This is an open access article under the terms of the Creative Commons Attribution-NonCommercialNoDerivs License, which permits use and distribution in any medium, provided the original work is properly cited, the use is non-commercial and no modifications or adaptations are made. 
oral hygiene. The most evident signs of gingivitis are gingival inflammation and bleeding (3). The principal risk factor for gingivitis is the bacterial plaque which forms on the gingiva and tooth surfaces (2-4). Plaque control is the key to prevention and the first step in the treatment of periodontal diseases $(5,6)$, which consists of mechanical and chemical methods. The chemical method is more common and cost-effective (5); however, since it relies on the individual's capabilities, it is not always reliable. Therefore, chemical methods are necessary as adjunctive methods for proper control of plaque and gingivitis $(4,5)$. Currently, a large number of mouthwashes are available to this end, of which chlorhexidine (CHX) has been introduced as the most effective chemical agent to control plaque $(2,4$ 6). The high efficacy of CHX, a cationic bisbiguanide molecule, has been attributed to its high substantivity in the oral cavity and also its bactericidal and bacteriostatic activities (7). CHX does not cause systemic poisoning due to its low absorption from the digestive system (8). It is rapidly adsorbed, due to its positive charge, to surfaces with a negative charge such as intraoral mucous membranes, salivary pellicles on tooth surfaces, tongue, salivary proteins, biofilm components, including bacteria, extracellular polysaccharides and glycoproteins $(1,9,10)$. After it is adsorbed to the cell membrane of bacteria, it damages the membrane, resulting in leakage of intracellular components. CHX adsorbed to various surfaces is gradually released into the oral cavity. Slow release of CHX results in prolongation of its antibacterial activity in the oral cavity for several hours, which depends on different factors such as dose, intervals of use of the mouthwash, temperature, presence of tooth or prostheses and the salivary $\mathrm{pH}$ (1). Use of CHX mouthwash results in some side effects such as brown discoloration of teeth, some restorative materials and the tongue $(2,4,8)$, interference with taste perception and a dryness and burning sensation in the oral cavity (1). In relation to anti-plaque and anti-gingivitis properties, some studies have been carried out on mouthwashes containing essential oil (EO) (for example: thymol, menthol, eucalyptol and methyl salicylate) and the results have shown that these mouthwashes, too, are effective in the treatment of gingivitis (11-14). A mouthwash has been manufactured in Iran, called Vi-one $(0.2 \%$ CHX, without alcohol) and the manufacturer claims that it has the highest efficacy in preventing formation of bacterial plaque and prevents gingival inflammation and bleeding, with minimum side effects (15). The manufacturer claims that this mouthwash exhibits antimicrobial activity since it contains active components such as thymol $(0.020 \%)$, which is one of the most important ingredients of thyme, and promotes the effect of CHX on prevention of plaque formation; in addition, it has a pleasant taste and exhibits antioxidative properties (15). Furthermore, it helps tooth remineralization because it has xylitol $(0.040 \%)$ in its composition, and decreases the side effects of $0.2 \%$ CHX (15). The chemical composition of mouthwashes affects their efficacy (16); therefore, the antibacterial effect of CHX can differ between different brands. The present study was undertaken to determine the efficacy and side effects of CHX mouthwash in combination with thymol since no study was found on the effects of such a mouthwash.

\section{Material and Methods}

\subsection{Trial design and participants}

This study was a randomized clinical trial that was conducted from December 22, 2015 to June 22, 2016. This double-blind randomized clinical trial study was performed on 60 patients, who referred to the Department of Periodontology, Faculty of Dentistry, Babol University of Medical Science.

\subsection{Selection criteria}

The inclusion criteria for participation in this study were: patients who were able to cooperate with similar plaque index (Silness and Loe), having chronic moderate-severe plaque induced gingivitis, being the age of 25 to 50 years old. The exclusion criteria were as follows: patients with history of systemic disease that could influence periodontium, patients who smoked, patients who were under periodontal treatment during the past 6 months and patients who received antibiotics during the last month.

\subsection{Interventions}

Eligible patients were included in the present study after the study procedures were explained to them by the operator and after they signed informed consent forms. Patients in each group underwent scaling, root planning and polishing, then were educated about BASS-Method brushing and recommended the Oral-B toothbrush and Pooneh toothpaste. Patients were randomly given a bottle of the mouthwash that each bottle contained Vi-one $0.2 \%$ CHX or Behsa $0.2 \%$ CHX. The two groups were asked to rinse their mouths for 60 seconds twice a day, once in the morning and once at night, after brushing their teeth and not to rinse their mouths for two hours after using the respective mouthwashes and abstain from eating; they were expected to continue these procedures for 2 weeks.

\subsection{Outcomes}

The following clinical parameters were recorded at baseline and 2 weeks later in Ramfjord teeth: Plaque index (PI, Silness and Loe), Gingival index (GI, Silness and Loe), Bleeding index (BI, Barnett), and Stain index (SI, Green and Vermillion). 


\subsection{Sample size}

The sample size was calculated to be 60 patients. This sample size was calculated based on the results of previous study (5) by assuming the test power of $80 \%$ and a confidence level of $95 \%$.

\subsection{Randomization and blinding}

Randomization was done by one of the researchers who did not have a role in the treatment of the participants. The randomization sequence was computer-generated, with the randomization itself conducted through IBM $\odot$ SPSS $\odot$ Statistics version 21 (IBM $\odot$ Corp., Armonk, NY, USA) (random number generation). After coding identical bottles containing the mouthwashes, each patient was randomly given a bottle of the mouthwash by an operator who was blinded to the type of the mouthwash in each bottle. Each bottle contained Vi-one $0.2 \%$ CHX or Behsa $0.2 \%$ CHX.

\subsection{Statistical methods}

Data analysis was conducted using SPSS version 21 software. We used independent-samples t-test and pairedsamples t-test to analyze the primary and secondary outcomes. The statistical difference was significant at $\mathrm{p}<0.05$. Also, before performing the statistical analyses, the normality of the variables' distribution was examined using the K-S test.

\subsection{Research ethics}

The proposal for this thesis research was presented to the Ethics Committee of Babol University of Medical Sciences (MUBABOL.REC.1394.188). Participation in this study was voluntary and patients could leave the study in each stage. Indeed, in this study, for ethical considerations, the participants were informed about the objective and nature of the study, and each participant provided her written consent in her native language (Persian) prior to the study. Also, we were committed to keeping all of the participants' information confidential. Mouthwash that had been given to patients was standard, was approved by the Ministry of Health and is available in pharmacies across the country.

\section{Results}

The mean PI and GI decreased significantly after intervention in both groups, but the mean difference of the group that rinsed Vi-one was significantly higher than the Behsa group $(\mathrm{p}<0.001, \mathrm{p}=0.021$ respectively) (Table 1$)$. According to the table of gingival index, before the study, all patients who used Vi-one mouthwash had moderate gingivitis and after the study, all of them had mild gingivitis. Before the study, 29 patients who used Behsa mouthwash had moderate gingivitis and one patient had severe gingivitis, and after the study, 27 patients had mild and three of them had moderate gingivitis. Mean values (standard deviations) of the percentage of bleeding for the Vi-one mouthwash initially and finally were 83.75 (28.70) and 19.44 (10.40), respectively, and for the Behsa mouthwash were 89.31 (26.90) and 25.97 (14.46), respectively. Analysis of this data show that the mean BI in the Vi-one group decreased more than that in the Behsa group, but there were no significant differences between the two groups $(\mathrm{p}=0.879)$. Mean values (standard deviations) of the percentage of staining for the Vi-one mouthwash initially and finally were 41.81 (18.71) and 107.50 (27.23), respectively, and for the Behsa mouthwash were 45.56 (21.99) and 109.58 (27.83), respectively. Analysis of this data show that the SI increased in both groups, with greater increase in the Vi-one group, but no statistically significant differences were observed between the two groups $(\mathrm{p}=0.754)$.

Table 1. Mean and mean difference of PI and GI of two groups

\begin{tabular}{|l|l|l|l|l|l|l|}
\hline Variable & \multicolumn{2}{l|}{ PI $($ Mean \pm SD $)$} & \multicolumn{2}{l|}{ GI $($ Mean \pm SD $)$} \\
\hline Type of mouthwash & Before & After & Difference & Before & After & Difference \\
\hline Vi-one & $1.658 \pm 0.062$ & $0.515 \pm 0.143$ & $1.143 \pm 0.161$ & $1.533 \pm 0.209$ & $0.608 \pm 0.184$ & $0.925 \pm 0.225$ \\
\hline Behsa & $1.65 \pm 0.047$ & $0.820 \pm 0.122$ & $0.830 \pm 0.120$ & $1.504 \pm 0.244$ & $0.738 \pm 0.198$ & $0.765 \pm 0.292$ \\
\hline
\end{tabular}

\section{Discussion}

The present study was carried out to clinically evaluate the efficacy of Vi-one CHX mouthwash, as an adjunct in the treatment of dental plaque-induced gingivitis. In this study, the mean difference of PI and GI in the group that rinsed Vi-one was significantly higher than the Behsa group, which is not similar to the result of previous studies. Papaioannou et al. carried out a study on new $0.2 \% \mathrm{CHX}$ without alcohol and conventional alcohol-containing $0.2 \%$ $\mathrm{CHX}$, and reported that mean values of PI increased similarly for both solutions; however, these differences between initial and final values were statistically significant only for CHX without alcohol mouthwash. Similarly, the mean values for the GI showed small increases, but with no statistically significant differences between them (17). Jose et 
al. too, reported that $0.2 \%$ CHX with and without alcohol, in association with brushing with a standard fluoridecontaining toothpaste, decreased plaque formation and gingival inflammation compared to the situation in which the teeth were brushed only, with no significant difference between the two groups (1). Todkar et al. reported that $0.2 \%$ CHX, with and without alcohol, significantly decreased PI and GI compared to placebo; however, $0.2 \%$ CHX without alcohol resulted in more clinical changes in GI compared to $0.2 \% \mathrm{CHX}$ containing alcohol (18). Charles et al. carried out a 6-month clinical trial and reported that $0.12 \%$ CHX mouthwash and a mouthwash containing EO significantly decreased PI and GI compared to a mouthwash containing 5\% hydroalcohol (control group), with no significant differences between the two groups (11). Pan et al. evaluated the efficacy of 5 different mouthwashes and reported higher anti-plaque activity of $0.12 \%$ CHX mouthwash and a mouthwash containing EO $(0.064 \%$ thymol, $0.092 \%$ eucalyptol, $0.060 \%$ methyl salicylate and $0.042 \%$ menthol) compared to mouthwashes containing $0.05 \%$ cetyl pyridinium chloride (CPC) and $0.05 \% \mathrm{CPC} / 0.05 \% \mathrm{CHX}$ and a mouthwash containing amine fluoride/stannous fluoride (13). Osso et al. reported high clinical efficacy of mouthwash containing $0.12 \%$ CHX and mouthwash containing EO (menthol, thymol and eucalyptol) against gingivitis and plaque formation compared to other mouthwashes evaluated in their study (12). Neely reported higher efficacy for CHX mouthwash in decreasing dental plaque compared to a mouthwash containing EO at short term $(<4$ weeks) and long term ( $>4$ weeks). These two mouthwashes exhibited similar effects on containing gingival inflammation at long term (19). Based on a two-week study, Parikh-Das et al. reported that an EO mouthwash was more effective in preventing plaque formation and improving gingivitis compared to a mouthwash containing $0.075 \% \mathrm{CPC}$ (14). As mentioned in the first part of "Discussion" in the present study, Vi-one mouthwash (CHX/ thymol/ alcohol-free) was more effective than Behsa mouthwash (CHX/ alcohol) in decreasing plaque and gingival inflammation. Studies by Jose and Todkar and Papaioannou et al. showed no significant differences between CHX mouthwashes with and without alcohol in decreasing PI and GI, which is different from the results of the present study. In this context, only in the study by Papaioannou et al, differences between initial and final values were statistically significant only for CHX without alcohol mouthwash and in the study by Todkar et al., CHX mouthwash without alcohol exhibited better clinical efficacy in decreasing GI compared to alcohol-containing CHX mouthwash, consistent with the results of the present study. Studies by Charles, Pan, Osso, Neely and Parikh-Das showed a lower efficacy of EO mouthwash in decreasing PI and gingivitis compared to CHX mouthwash; however, its efficacy was higher than that of CPC mouthwash. Therefore, the differences between the results of the present study and other studies might be attributed to thymol in addition to CHX in the composition of Vi-one mouthwash and their possible synergistic effect. Thymol is a phenolic compound, which is extracted from thyme $(20,21)$. It exhibits antimicrobial and antifungal activities in a mechanism similar to that of CHX. Thymol inhibits the growth of many microorganisms by protein denaturation and destruction of cell membrane (21). It has antioxidative and anti-inflammatory properties (22) and its extract has been commercially used as an ingredient in the composition of mouthwash in recent years (23). Botelho et al. based on their study on the antimicrobial activity of thymol against oral pathogens, reported that thymol was effective in inhabiting the growth of oral pathogens; therefore, it can be useful for the preservation of oral hygiene (24). Didry et al. reported that thymol can be used for the treatment of oral infections alone or in combination with eugenol or carvacrol (25). Takeuchi et al. evaluated the effect of CHX/thymol varnish and fluoride varnish on the formation of dental biofilm in vitro and reported that CHX/thymol varnish inhibited biofilm formation but fluoride varnish did not exhibit such capability (26). Yucel-Lidberg et al. reported that in patients with chronic gingival inflammation, local use of CHX /thymol varnish was useful (27). Filoche et al. reported that the amount of chlorhexidine necessary to achieve an equivalent growth inhibition against Lactobacillus plantarum and Streptococcus mutans was several times lower when it was combined with EO compared to CHX alone; therefore, they concluded that EO might have a role in the development of new anti-caries treatments (28). In the present study, in both groups gingival bleeding decreased and tooth staining increased, with no significant differences between the two groups. The results of a study by Papaioannou et al. (17) in relation to staining, were consistent with those of the present study. The results of the present study were consistent with those of studies by Jose et al. on $0.2 \%$ CHX mouthwash with and without alcohol (1), by Leyes Borrajo et al. on $0.12 \%$ CHX, $0.05 \%$ sodium fluoride, $11 \%$ ethanol and the same mouthwash without alcohol (6) in relation to B I Charles et al. who reported that $0.12 \%$ CHX mouthwash resulted in significantly more calculus and stains compared to EO mouthwash and the control group (11). Neely reported, based on a review study, that formation of calculus and stain is higher with the use of CHX mouthwash compared to the use of an EO mouthwash (19). Based on the results of the present study and those of the studies above, it can be concluded that the presence of thymol in addition to CHX in Vi-one mouthwash does not possibly have a synergistic effect on BI and SI. 


\section{Conclusions}

In brief, results of this study showed that Vi-one mouthwash is effective in reducing the Plaque Index and Gingival Index. Based on this result, we conclude that Vi-one mouthwash can be offered to patients with dental plaqueinduced gingivitis, but we think that further studies are necessary to support this conclusion with stronger evidence.

\section{Acknowledgments:}

We appreciate the Professors of the Department of Periodontology, Babol University of Medical Sciences, especially Ms. Faranak Farsam for her cooperation in the implementation of the study, and we sincerely appreciate all of the patients who participated in the study.

\section{Trial Registration:}

The trial was registered at the Iranian Registry of Clinical Trials (http://www.irct.ir) with the Irct ID: IRCT201602231760N45.

\section{Funding:}

This study was a part of thesis and research project (Grant No: 9440921) supported and funded by Babol University of Medical Sciences. The authors received no financial support from Behsa pharmaceutical company (Behsa mouthwash manufacturer) and Rojin cosmetic laboratory (Vi-one mouthwash manufacturer) for this study.

\section{Conflict of Interest:}

There is no conflict of interest to be declared.

\section{Authors' contributions:}

All authors contributed to this project and article equally. All authors read and approved the final manuscript.

\section{References:}

1) Jose A, Butler A, Payne D, Maclure R, Rimmer P, Bosma ML. A randomised clinical study to evaluate the efficacy of alcohol-free or alcohol-containing mouthrinses with chlorhexidine on gingival bleeding. British dental journal. 2015;219(3):125-30. Doi: 10.1038/sj.bdj.2015.592, PMID:26271869.

2) Kumar S, Patel S, Tadakamadla J, Tibdewal H, Duraiswamy P, Kulkarni S. Effectiveness of a mouthrinse containing active ingredients in addition to chlorhexidine and triclosan compared with chlorhexidine and triclosan rinses on plaque, gingivitis, supragingival calculus and extrinsic staining. Int J Dent Hyg. 2013;11(1):35-40. Doi: 10.1111/j.1601-5037.2012.00560.x, PMID:22672130.

3) Aspalli S, Shetty VS, Devarathnamma MV, Nagappa G, Archana D, Parab P. Evaluation of antiplaque and antigingivitis effect of herbal mouthwash in treatment of plaque induced gingivitis: A randomized, clinical trial. Journal of Indian Society of Periodontology. 2014;18(1):48-52. Doi: 10.4103/0972-124X.128208, PMID: 24744544.

4) Bhate D, Jain S, Kale R, Muglikar S. The comparative effects of $0.12 \%$ chlorhexidine and herbal oral rinse on dental plaque-induced gingivitis: A randomized clinical trial. Journal of Indian Society of Periodontology. 2015;19(4):393-5. Doi: 10.4103/0972-124X.153478, PMID: 26392686.

5) Najafi MH, Taheri M, Mokhtari MR, Forouzanfar A, Farazi F, Mirzaee M, et al. Comparative study of $0.2 \%$ and $0.12 \%$ digluconate chlorhexidine mouth rinses on the level of dental staining and gingival indices. Dental research journal. 2012;9(3):305-8. PMID: 23087736.

6) Leyes Borrajo JL, Garcia VL, Lopez CG, Rodriguez-Nunez I, Garcia FM, Gallas TM. Efficacy of chlorhexidine mouthrinses with and without alcohol: a clinical study. J Periodontol. 2002;73(3):317-21. Doi: 10.1902/jop.2002.73.3.317, PMID: 11924590.

7) Quirynen M, Avontroodt P, Peeters W, Pauwels M, Coucke W, van Steenberghe D. Effect of different chlorhexidine formulations in mouthrinses on de novo plaque formation. Journal of clinical periodontology. 2001;28(12):1127-36. PMID: 11737510.

8) Van Strydonck DA, Slot DE, Van der Velden U, Van der Weijden F. Effect of a chlorhexidine mouthrinse on plaque, gingival inflammation and staining in gingivitis patients: a systematic review. Journal of clinical periodontology. 2012;39(11):1042-55. Doi: 10.1111/j.1600-051X.2012.01883.x, PMID: 22957711.

9) Mor-Reinoso C, Pascual A, Nart J, Quirynen M. Inhibition of de novo plaque growth by a new $0.03 \%$ chlorhexidine mouth rinse formulation applying a non-brushing model: a randomized, double blind clinical trial. Clinical oral investigations. 2016;20(7):1459-67. Doi: 10.1007/s00784-015-1625-y, PMID: 26475499. 
10) Zanatta FB, Antoniazzi RP, Rosing CK. Staining and calculus formation after $0.12 \%$ chlorhexidine rinses in plaque-free and plaque covered surfaces: a randomized trial. Journal of applied oral science : revista FOB. 2010;18(5):515-21. PMID: 21085810.

11) Charles $\mathrm{CH}$, Mostler KM, Bartels LL, Mankodi SM. Comparative antiplaque and antigingivitis effectiveness of a chlorhexidine and an essential oil mouthrinse: 6-month clinical trial. Journal of clinical periodontology. 2004;31(10):878-84. Doi: 10.1111/j.1600-051X.2004.00578.x, PMID:15367192.

12) Osso D, Kanani N. Antiseptic mouth rinses: an update on comparative effectiveness, risks and recommendations. Journal of dental hygiene : JDH / American Dental Hygienists' Association. 2013;87(1):10-8. PMID: 23433693.

13) Pan PC, Harper S, Ricci-Nittel D, Lux R, Shi W. In-vitro evidence for efficacy of antimicrobial mouthrinses. Journal of dentistry. 2010;38 Suppl 1:S16-20. Doi: 10.1016/S0300-5712(10)70006-3, PMID: 20621239.

14) Parikh-Das AM, Sharma NC, Du Q, Charles CA. Superiority of essential oils versus $0.075 \%$ CPCcontaining mouthrinse: a two-week randomized clinical trial. The Journal of clinical dentistry. 2013;24(3):94-9. PMID: 24660271.

15) www.rojin-cosmetic.ir

16) Ghasemi M, Janab H, Valaei N. Anti-Plaque Efficacy of Chlorhexidine Mouthrinses With and without Alcohol. Res Dent Sci. 2014;10(4):211-7.

17) Papaioannou W, Vassilopoulos S, Vrotsos I, Margaritis V, Panis V. A comparison of a new alcohol-free $0.2 \%$ chlorhexidine oral rinse to an established $0.2 \%$ chlorhexidine rinse with alcohol for the control of dental plaque accumulation. Int J Dent Hyg. 2015. Doi: 10.1111/idh.12182, PMID: 26449740.

18) Todkar R, Sheikh S, Byakod G, Muglikar S. Efficacy of chlorhexidine mouthrinses with and without alcohol - a clinical study. Oral health \& preventive dentistry. 2012;10(3):291-6. PMID: 23094273.

19) Neely AL. Essential oil mouthwash (EOMW) may be equivalent to chlorhexidine (CHX) for long-term control of gingival inflammation but $\mathrm{CHX}$ appears to perform better than EOMW in plaque control. The journal of evidence-based dental practice. 2012;12(3 Suppl):69-72. Doi: 10.1016/S1532-3382(12)70017-9, PMID: 23253835.

20) Shu C, Sun L, Zhang W. Thymol has antifungal activity against Candida albicans during infection and maintains the innate immune response required for function of the p38 MAPK signaling pathway in Caenorhabditis elegans. Immunologic research. 2016;64(4):1013-24. Doi: 10.1007/s12026-016-8785-y, PMID: 26783030.

21) Cosyn J, Wyn I, De Rouck T, Moradi Sabzevar M. Clinical benefits of subgingival chlorhexidine varnish application as an adjunct to same-day full-mouth root planing: a pilot study. J Periodontol. 2006;77(6):1074-9. Doi: 10.1902/jop.2006.050220, PMID: 16734584.

22) Marchese A, Orhan IE, Daglia M, Barbieri R, Di Lorenzo A, Nabavi SF, et al. Antibacterial and antifungal activities of thymol: A brief review of the literature. Food chemistry. 2016;210:402-14. Doi: 10.1016/j.foodchem.2016.04.111, PMID: 27211664.

23) Walsh SE, Maillard JY, Russell AD, Catrenich CE, Charbonneau DL, Bartolo RG. Activity and mechanisms of action of selected biocidal agents on Gram-positive and -negative bacteria. Journal of applied microbiology. 2003;94(2):240-7. PMID: 12534815.

24) Botelho MA, Nogueira NA, Bastos GM, Fonseca SG, Lemos TL, Matos FJ, et al. Antimicrobial activity of the essential oil from Lippia sidoides, carvacrol and thymol against oral pathogens. Brazilian journal of medical and biological research $=$ Revista brasileira de pesquisas medicas e biologicas / Sociedade Brasileira de Biofisica [et al]. 2007;40(3):349-56. PMID: 17334532.

25) Didry N, Dubreuil L, Pinkas M. Activity of thymol, carvacrol, cinnamaldehyde and eugenol on oral bacteria. Pharmaceutica acta Helvetiae. 1994;69(1):25-8. PMID: 7938073.

26) Takeuchi Y, Guggenheim B, Filieri A, Baehni P. Effect of chlorhexidine/thymol and fluoride varnishes on dental biofilm formation in vitro. European journal of oral sciences. 2007;115(6):468-72. Doi: 10.1111/j.1600-0722.2007.00493.x, PMID: 18028054.

27) Yucel-Lindberg T, Twetman S, Skold-Larsson K, Modeer T. Effect of an antibacterial dental varnish on the levels of prostanoids, leukotriene B4, and interleukin-1 beta in gingival crevicular fluid. Acta odontologica Scandinavica. 1999;57(1):23-7. PMID: 10207532.

28) Filoche SK, Soma K, Sissons CH. Antimicrobial effects of essential oils in combination with chlorhexidine digluconate. Oral microbiology and immunology. 2005;20(4):221-5. Doi: 10.1111/j.1399302X.2005.00216.x, PMID: 15943766 